Linköping Studies in Science and Technology.

Dissertations, No. 1740

\title{
High order summation-by-parts methods in time and space
}

\author{
Tomas Lundquist
}

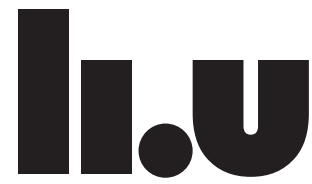

LINKÖPING UNIVERSITY

Department of Mathematics, Division of Computational Mathematics Linköping University, SE-581 83 Linköping, Sweden

Linköping 2016 
Linköping Studies in Science and Technology. Dissertations, No. 1740 High order summation-by-parts methods in time and space Copyright (c) Tomas Lundquist, 2016

Division of Computational Mathematics

Department of Mathematics

Linköping University

SE-581 83, Linköping, Sweden

tomas.lundquist@liu.se

www.liu.se/mai/ms

Typeset by the author in $\mathrm{A}_{\mathrm{E}} \mathrm{X} 2 \mathrm{e}$ documentation system.

ISSN 0345-7524

ISBN 978-91-7685-837-0

Printed by LiU-Tryck, Linköping, Sweden 2016 


\section{Abstract}

This thesis develops the methodology for solving initial boundary value problems with the use of summation-by-parts discretizations. The combination of high orders of accuracy and a systematic approach to construct provably stable boundary and interface procedures makes this methodology especially suitable for scientific computations with high demands on efficiency and robustness. Most classes of high order methods can be applied in a way that satisfies a summation-by-parts rule. These include, but are not limited to, finite difference, spectral and nodal discontinuous Galerkin methods.

In the first part of this thesis, the summation-by-parts methodology is extended to the time domain, enabling fully discrete formulations with superior stability properties. The resulting time discretization technique is closely related to fully implicit Runge-Kutta methods, and may alternatively be formulated as either a global method or as a family of multi-stage methods. Both first and second order derivatives in time are considered. In the latter case also including mixed initial and boundary conditions (i.e. conditions involving derivatives in both space and time).

The second part of the thesis deals with summation-by-parts discretizations on multi-block and hybrid meshes. A new formulation of general multi-block couplings in several dimensions is presented and analyzed. It collects all multiblock, multi-element and hybrid summation-by-parts schemes into a single compact framework. The new framework includes a generalized description of nonconforming interfaces based on so called summation-by-parts preserving interpolation operators, for which a new theoretical accuracy result is presented. 



\section{Sammanfattning på svenska}

Denna avhandling utgörs av en serie artiklar inom området numerisk lösning av tidsberoende partiella differentialekvationer. Sådana utnyttjas vid modellering av många skilda fenomen inom främst naturvetenskapliga och tekniska tillämpningar, såsom flödesdynamik, elektromagnetism eller strukturmekanik. De matematiska modellerna löses med hjälp av diskreta approximationer av de styrande partiella differentialekvationerna. Denna procedur är ofta både teoretiskt utmanande och resurskrävande i form av datorkapacitet. Utvecklandet av nya robusta och effektiva numeriska metoder för partiella differentialekvationer är därför av stor vikt, och utgör sedan lång tid ett omfattande självständigt forskningsområde.

Vid numerisk lösning är stabilitet ett nödvändigt kriterium för att garantera en konvergent lösning. Stabilitet är framförallt svårt att uppnå vid ränderna till ett beräkningsområde, samt vid gränsytor mellan områden där olika typer av diskretiseringsscheman används. Särskilt gäller detta för metoder av hög noggrannhetsordning, vilka är önskvärda ur effektivitetssynpunkt. Stabil tidsintegrering kan i vissa fall också vara en utmaning, t.ex. om det rumsdiskretiserade problemet innehåller oupplösta fenomen eller lokalt förfinade områden. Genom att använda diskretiseringsscheman av s.k. partiell summationstyp (eng. "summation-by-parts", SBP) kan numerisk stabilitet uppnås på ett systematiskt vis som går att styrka med matematiska bevis.

Denna avhandling bidrar dels med att utvidga den partiella summationstekniken till tidsdomänen, vilket möjliggör fullt diskreta formuleringar inom ett och samma ramverk med utmärkta stabilitetsegenskaper. Både första och andra ordningens derivator i tiden kan diskretiseras på detta vis, och i det senare fallet kan även blandade randvillkor med både rums- och tidsderivator behandlas. Det andra huvudsakliga bidraget gäller s.k. hybridformuleringar, dvs där olika diskretiseringsscheman används i olika delar av beräkningsområdet. Detta kan t.ex. göras av effektivitetsskäl eller för ökad geometrisk flexibilitet. Ett nytt generellt ramverk för denna typ av formuleringar i flera rumsdimensioner har presenterats. Det nya ramverket innefattar en generaliserad beskrivning av så kallade SBP-bevarande interpolationsscheman, vilket möjliggör stabila kopplingar mellan olika områden. I samband med detta har ett nytt teoretiskt noggrannhetsresultat bevisats för SBP-bevarande scheman. 



\section{Acknowledgements}

First of all I want to sincerely thank my supervisor Prof. Jan Nordström for his invaluable support and guidance during my years at LiU. The level of commitment from him as a mentor and in providing feedback has been truly outstanding, and I would not have come as far as I have in my research without it.

I would also like to thank my colleagues in the research group for their moral and professional support. They have all contributed to making my time at the division more productive and enjoyable. Among them I would especially like to mention Viktor Linders for the many stimulating discussions on various research topics, and Ossian O'Reilly for inspirational and productive collaboration during his visits in Linköping. I also give special thanks to Peter Eliasson and Andrea Ruggiu for our respective research collaboration, and to Fredrik Berntsson and Marco Kupiainen for generous sharing of their expert knowledge whenever I have needed it.

Finally I want to thank my family and all of my friends, both from work and from outside, for all their love and support.

This work has been partially funded by the Swedish Research Council (VR). 



\section{List of Papers}

This thesis is based on the following papers, which will be referred to in the text by their roman numerals.

I. J. Nordström and T. Lundquist, Summation-by-parts in time, Journal of Computational Physics 251 (2013) 487-499.

II. T. Lundquist and J. Nordström, The SBP-SAT technique for initial value problems, Journal of Computational Physics 270 (2014) 84-104.

III. T. Lundquist and J. Nordström, Efficient fully discrete summation-byparts schemes for unsteady flow problems, BIT Numerical Mathematics (2015) 1-16.

IV. J. Nordström and T. Lundquist, Summation-by-parts in time: the second derivative, accepted in: SIAM Journal on Scientific Computing (2016).

V. T. Lundquist and J. Nordström, On the suboptimal accuracy of summationby-parts schemes with non-conforming block interfaces, Submitted (2016).

VI. T. Lundquist and J. Nordström, An energy stable summation-by-parts formulation for general multi-block and hybrid meshes, Submitted (2016).

I have contributed to the works listed above by deriving most of the novel theoretical results, as well as by producing all numerical calculations. The manuscripts have been written by myself in their entirety (although with generous editorial support from my main supervisor prof. Jan Nordström), with the exception of paper I and paper IV which were written jointly together with the first author. 



\section{Contents}

Abstract i

Sammanfattning på svenska iii

Acknowledgements $\quad$ v

List of Papers vii

1 Introduction 1

2 Summation-by-parts operators $\quad 3$

2.1 A motivating example . . . . . . . . . . . . . . 3

2.2 Difference formulations . . . . . . . . . . . . . . 4

2.3 Accuracy ....................... 5

3 The simultaneous-approximation-

$\begin{array}{ll}\text { term technique } & \mathbf{7}\end{array}$

3.1 Weak boundary conditions . . . . . . . . . . . . 7

3.2 Weak initial conditions . . . . . . . . . . . . . . 8

3.3 Weak interface conditions . . . . . . . . . . . . . . . . 10

3.3.1 Conservation and stability ............ 11

4 Summary of papers 13

4.1 Paper I . . . . . . . . . . . . . . . . . . . . . 13

4.2 Paper II . . . . . . . . . . . . . . . . . . . . . . 14

4.3 Paper III . . . . . . . . . . . . . . . . . . . 15

4.4 Paper IV . . . . . . . . . . . . . . . . . 16

4.5 Paper V . . . . . . . . . . . . . . . . . . . 17

4.6 Paper VI . . . . . . . . . . . . . . . . . . 18

$\begin{array}{ll}\text { References } & 20\end{array}$ 


\section{INCLUDED PAPERS}

I. Summation-by-parts in time . . . . . . . . . . . . . . . 23

II. The SBP-SAT technique for initial value problems . . . . . . . . . . 39

III. Efficient fully discrete summation-by-parts schemes for unsteady flow problems ................... 61

IV. Summation-by-parts in time: the second derivative . . . . . . . . . 79

V. On the suboptimal accuracy of summation-by-parts schemes for non-conforming block interfaces . . . . . . . . . . . 107

VI. An energy stable summation-by-parts formulation for general multiblock and hybrid meshes . . . . . . . . . . . . . . . . . . 119 


\section{Introduction}

Time dependent partial differential equations (PDE) can be used to model a wide range of natural phenomena of scientific and technological interest. Important areas of applications include e.g. solid and fluid dynamics and wave propagation problems. In most cases the models can only be solved using numerical methods, by approximating the governing equations at discrete points in space and time. This poses challenges both from a theoretical point of view as well as by requiring extensive computer resources. The rapid advances in computer technology over the past decades has greatly extended the number of complex phenomena from science and engineering feasible for numerical simulation. This development has increased rather than decreased the demand for efficient and robust numerical mehods.

The first criterion for a successful numerical simulation, apart from correctly representing the physics involved, is that the model itself should be well-posed in a mathematical sense. Once this has been achieved, a discrete equation system approximating the governing equations is formulated. In order to guarantee a convergent numerical solution, the discretization must also prevent non-physical growth. This property is generally refered to as stability, and is difficult to achive close to the boundaries of the simulation domain and along numerical interfaces between different types of discretizations. This is especially true for methods with high orders of accuracy, which is desirable for efficiency.

The idea behind using discrete differential operators on summation-by-parts (SBP) form is to mimic key features of the underlying PDE that can be used to prove stability. Although originally developed for high order finite fifference methods (HOFDM) beginning in [8], the SBP framework has also been shown in later years to incorporate a number of other types of methods as well. These include finite volume (FVM), spectral and discontinuous Galerkin (dG) methods. For optimal utility in terms of stability, SBP operators can be augmented with weak boundary and interface conditions using the simultaneous-approximationterm (SAT) technique, first introduced in [3]. The combination of these two concepts leads to the so-called SBP-SAT technique, which is an ideally suited framework in which to construct provably stable and high order accurate discretizations.

In this thesis we extend the SBP-SAT framework to the time domain, which leads to a set of fully implicit time marching schemes with optimal stability 
properties. The theory is developed and tested for both first and second derivatives in time, also incorporating mixed boundary conditions with derivatives in both time and space. The second contribution from this thesis consists of developing a new framework for general SBP-SAT discretizations on multiple domains. New theoretical results involving accuracy, conservation and stability are formulated within this framework. Based on these, a generalized description of so-called SBP preserving interpolation operators for non-conforming interface treatments is presented. A new theoretical accuracy result for the previously known SBP preserving interpolation operators is also derived.

The notation used in the summary of the papers may deviate from the one used within the papers themselves. This choice was made in order to keep the notation as consistent as possible throughout the introductory chapters of this thesis. 


\section{Summation-by-parts operators}

One of the key features of the SBP-SAT technique is the use of discrete first derivative operators on summation-by-parts (SBP) form. The discrete operator is constructed to mimic the integration-by-parts (IBP) rule of the continuous derivative. IBP is central for proving well-posedness of initial boundary value problems with the energy method. In one space dimension, the integration-byparts rule is

$$
\int_{0}^{1} \phi \psi_{x}=\left.(\phi \psi)\right|_{0} ^{1}-\int_{0}^{1} \phi_{x} \psi .
$$

Higher order discrete derivatives can be generated by simply applying a first order SBP operator repeatedly, but it is also possible to develop compact high order derivatives on SBP form directly. Multi-dimensional discretizations can likewise be formulated either by extending the one-dimensional operators with the use of Kronecker products, or alternatively be constructed directly for the multi-dimensional grid.

Before introducing the concept of SBP operators, we start with a motivating example from the finite element method, similar to the one used in [8].

\subsection{A motivating example}

As a model problem, consider the advection equation with unit wave speed in one dimension, given by

$$
\begin{aligned}
u_{t}+u_{x} & =0, & & x \in(0,1), \quad t>0 \\
u & =g(t), & & x=0 \\
u & =f(x), & & t=0 .
\end{aligned}
$$

An energy estimate is obtained by multiplying the equation with $u$ and integrating in space. The IBP rule (2.1) then leads to

$$
\|u\|_{t}^{2}=g(t)^{2}-u(1, t)^{2}
$$

where $\|u\|=\int_{0}^{1} u^{2} d x$ is the $L_{2}$ norm of the solution. 
A stable discretization of (2.2) can be formulated with the Galerkin method. Consider a discrete grid $\mathbf{x}=\left(0=x_{0}, x_{1}, \ldots, x_{N-1}, x_{N}=1\right)^{T}$ in space, and approximate $u$ with $v=\varphi^{T} \alpha$, where $\varphi=\varphi(x)$ is a vector of basis functions, and $\alpha=\alpha(t)$ is another vector containing the unknown coefficients. Inserting $v$ into (2.2) and multiplying with the basis functions yields, after integrating in space,

$$
\int_{0}^{1} \varphi\left(\varphi^{T} \alpha_{t}+\varphi_{x}^{T} \alpha\right) d x=0 .
$$

This system can be rewritten as

$$
P \alpha_{t}+Q \alpha=0
$$

where $P=\int_{0}^{1} \varphi \varphi^{T} d t$ is a symmetric and positive definite mass matrix, and $Q=\int_{0}^{1} \varphi \varphi_{x}^{T} d t$ satisfies $Q+Q^{T}=\left.\left(\varphi \varphi^{T}\right)\right|_{0} ^{1}$ due to $(2.1)$. The discete energy method is applied by multiplying the Galerkin approximation (2.4) with $\alpha^{T}$ and then adding the transpose. This yields the estimate

$$
\frac{\partial}{\partial t}\|\alpha\|_{P}^{2}=-\left.\left(\left(\varphi^{T} \alpha\right)^{2}\right)\right|_{0} ^{1}
$$

where the discrete $L_{2}$ norm is defined as $\|\alpha\|_{P}^{2}=\alpha^{T} P \alpha$. For homogeneous boundary conditions, the estimate (2.4) is automatically stable if the basis functions in $\varphi$ also satisfy homogeneous boundary conditions.

\subsection{Difference formulations}

As the next step, consider a Lagrange polynomial basis in (2.4). In this case the coefficients are given by $\alpha=U$, where $U=\left(U_{0}, \ldots, U_{N}\right)^{T}$ approximates the solution at the node point. The variational formulation (2.4) thus becomes

$$
P U_{t}+Q U=0
$$

Due to the properties of Lagrange polynomials, the boundary terms in the energy estimate $(2.5)$ now satisfy $Q+Q^{T}=\left.\left(\varphi \varphi^{T}\right)\right|_{0} ^{1}=\operatorname{diag}(-1,0, \ldots, 0,1)$. The properties of the discretization matrices in (2.6) can be summarized as

$$
P=P^{T}>0, \quad Q+Q^{T}=\operatorname{diag}(-1,0, \ldots, 0,1),
$$

where $P$ as before assumes the role of a discrete $L_{2}$ integration operator and norm. The discrete energy estimate (2.5) becomes

$$
\frac{\partial}{\partial t}\|U\|_{P}^{2}=U_{0}^{2}-U_{N}^{2}
$$

Now, consider an approximation to (2.2) on difference form, given by

$$
U_{t}+D U=0
$$


where $D$ is a discrete operator approximating the continuous derivative $\partial / \partial x$. The original idea in [8] was to construct operators based on high order finite difference stencils that satisfy the decomposition $D=P^{-1} Q$, where $P$ and $Q$ in turn satisfy the algebraic conditions given in (2.7) leading to the energy rate (2.8). More generally, the conditions in (2.7) lead to a discrete version of the integration-by-parts rule (2.1). Let $\Phi$ and $\Psi$ be two vectors of the same dimension as the grid vector $\mathbf{x}$. Then $(2.7)$ gives

$$
\Phi^{T} P(D \Psi)=\Phi_{N} \Psi_{N}-\Phi_{0} \Psi_{0}-(D \Phi)^{T} P \Psi .
$$

The discrete SBP operators thus mimics IBP, but do not include the boundary conditions. The full potential of the development of SBP finite difference operators could be realized with the SAT technique for imposing boundary conditions weakly in [3].

As an example, let $P=\Delta x \operatorname{diag}(1 / 2,1, \ldots, 1,1 / 2)$ represent the standard trapezoidal rule, and let

$$
Q=\left(\begin{array}{cccccc}
-\frac{1}{2} & \frac{1}{2} & & & & \\
-\frac{1}{2} & 0 & \frac{1}{2} & & & \\
& -\frac{1}{2} & 0 & \frac{1}{2} & & \\
& & \ddots & \ddots & \ddots & \\
& & & -\frac{1}{2} & 0 & \frac{1}{2} \\
& & & & -\frac{1}{2} & \frac{1}{2}
\end{array}\right)
$$

The resulting operator $D=P^{-1} Q$ consists of a repeated second order accurate stencil in the interior, which is completed by inserting first order one-sided differences at both boundaries. Higher order finite difference stencils on SBP form follow the same principle, but require more complicated boundary closures as well as higher order accurate $L_{2}$-integration operators $P$.

\subsection{Accuracy}

The accuracy conditions for a truncation error of order of $p$ are given from Taylor's formula as

$$
D \mathbf{x}^{j}=j \mathbf{x}^{j-1}, \quad j=0, \ldots, p,
$$

where $\mathbf{x}=\left(x_{0}, \ldots, x_{N}\right)^{T}$ is the grid vector, and $(\cdot)^{j}$ denotes elementwise exponentiation for $j=0,1,2, \ldots$ For SBP finite difference operators with diagonal norms, the order $p$ is restricted to half of that of the central finite difference stencil in the interior [8].

In [7], the accuracy conditions (2.12) were combined with the SBP property (3.1) to derive accuracy relations also for the discrete $L_{2}$ integration operators. For a diagonal $P$, these are given by

$$
\mathbf{1}^{T} P \mathbf{x}^{\nu-1}=\frac{x_{N}^{\nu}-x_{0}^{\nu}}{\nu}=\frac{1}{\nu}, \quad \nu=1, \ldots, 2 p .
$$


In general, the matrix $P$ needs to be diagonal in order to ensure stability on curvilinear grids and for problems with non-constant coefficients [15, 14]. For this reason, we will only consider diagonal norms $P$ in this thesis. We will use both (2.12) and (2.13) to derive new accuracy results. 


\section{The simultaneous-approximation- term technique}

So far we have only considered the summation-by-parts property (2.7) of discrete difference operators. For a stable discretization of the full problem (2.2), a careful imposition of the boundary condition is also needed. The SAT method for imposing boundary, interface and initial conditions weakly is ideal to use together with SBP operators.

The SAT technique is a generic method of imposing boundary, interface or initial conditions weakly. The conditions are not imposed exactly, but rather enforced through penalty terms. The goal with this approach is to construct energy estimates that proves stability of the resulting discretization. The classical Lax theorem of equivalence [9] states that stability is equivalent to convergence of the solution for a consistent scheme. For non-linear conservation laws, an additional property known as conservation is also needed to ensure convergence to the correct solution, as formulated in the Lax-Wendroff theorem [10].

\subsection{Weak boundary conditions}

As a first step to introduce the boundary condition in (2.2), we define restriction operators to the boundaries of the domain in the form of the two row vectors $e_{0}=(1,0, \ldots, 0)$ and $e_{N}=(0, \ldots, 0,1)$, so that

$$
U_{0}=e_{0} U, \quad U_{N}=e_{N} U .
$$

The property in (2.7) can now be reformulated slightly in the following way. A discrete first derivative SBP operator is defined by the decomposition $D=$ $P^{-1} Q$, where

$$
P=P^{T}>0, \quad Q+Q^{T}=-e_{0}^{T} e_{0}+e_{N}^{T} e_{N},
$$

and where $P$ is assumed to be diagonal. The complete discrete problem approximating (2.2) is now obtained by replacing the continuous derivative with the corresponding discrete ones using $D$, and by imposing the boundary condition weakly in the top row of the discrete system. The standard SBP-SAT 
formulation for (2.2) yields

$$
U_{t}+D U=P^{-1} \sigma e_{0}^{T}\left(U_{0}-g(t)\right)
$$

where $\sigma$ is a so-called penalty coefficient. The linear system of ordinary differential equations to be solved can thus be written as

$$
U_{t}+\tilde{D} U=\tilde{g}(t)
$$

where

$$
\begin{aligned}
\tilde{D} & =P^{-1}\left(Q-\sigma e_{0}^{T} e_{0}\right) \\
\tilde{g} & =-P^{-1} \sigma e_{0}^{T} g .
\end{aligned}
$$

The modified operator $\tilde{D}$ can be thought of as the original difference operator $D$ augmented with a weak homogeneous boundary condition, while the addition of $\tilde{g}$ to the right hand side enforces the particular boundary data.

To verify that the implementation (3.3) is stable, consider

$$
P \tilde{D}+\tilde{D}^{T} P=Q+Q^{T}-2 \sigma e_{0}^{T} e_{0}=-(1+2 \sigma) e_{0}^{T} e_{0}+e_{N}^{T} e_{N} .
$$

The eigenvalues of $\tilde{D}$ have non-negative real parts if $\sigma \leq-1 / 2$, which implies that there is no non-physical growth present in the discrete system (3.3).

A clean discrete estimate mimicking (2.3) can be obtained with the specific choice $\sigma=-1$. Multiplying equation (3.3) with $U^{T} P$ and adding the transpose then yields

$$
\|U\|_{t}^{2}=-U^{T}\left(P \tilde{D}+\tilde{D}^{T} P\right) U+2 U_{0} g=-U_{0}^{2}-U_{N}^{2}+2 U_{0} g .
$$

After adding and subtracting $g^{2}$, and using (3.5), this gives the energy estimate

$$
\|U\|_{t}^{2}=g^{2}-U_{N}^{2}-\left(U_{0}-g\right)^{2},
$$

which mimics (2.3) with an additional damping term.

\subsection{Weak initial conditions}

In this thesis the SBP-SAT technique is extended to initial value problems as well. The most simple setting is given by a scalar model problem on the unit time interval. Consider

$$
\begin{aligned}
u_{t}+\lambda u & =0, \quad t \in(0,1) \\
u & =f, \quad t=0 .
\end{aligned}
$$

The energy method leads to the estimate

$$
u(1)^{2}+2 \lambda\|u\|^{2}=f^{2},
$$


where $\|u\|=\int_{0}^{1} u^{2} d t$. An SBP-SAT discretization on the discrete grid $\mathbf{t}=(0=$ $\left.t_{0}, t_{1}, \ldots, t_{N-1}, t_{N}=1\right)^{T}$ can now be formulated in analogy with (3.2), as

$$
(D+\lambda I) U=P^{-1} \sigma e_{0}^{T}\left(U_{0}-f\right),
$$

or equivalently, as

$$
(\tilde{D}+\lambda I) U=-P^{-1} \sigma e_{0}^{T} f .
$$

The discrete energy method for the choice $\sigma=-1$ yields the estimate

$$
U_{N}^{2}+2 \lambda\|U\|_{P}^{2}=f^{2}-\left(U_{0}-f\right)^{2}
$$

which mimics (3.8). Energy estimates like (3.10) can also be used to prove several formal stability properties of the SBP-SAT method in time. For instance, (3.10) itself implies $A$-stability of the method.

Combining the SBP-SAT approaches in time and space as outlined above leads to sharp fully discrete energy estimates. Consider the semi-discrete system (3.2) again. By using two different SBP operators $D_{x}$ and $D_{t}$ in space and time, respectively, the fully discrete SBP-SAT approximation with suitable penalty coefficients $(\sigma=-1)$ can be written as

$$
\left(D_{t} \otimes I_{x}\right) \mathbf{U}+\left(I_{t} \otimes D_{x}\right) \mathbf{U}=-\left(P_{t}^{-1} e_{0 t}^{T}\right) \otimes\left(\mathbf{U}_{0 t}-F\right)-\left(\mathbf{U}_{0 x}-G\right) \otimes\left(P_{x}^{-1} e_{0 x}^{T}\right),
$$

where $I_{t}$ and $I_{x}$ are unit matrices of the same dimensions as the corresponding SBP operators, and $\otimes$ denotes the Kronecker product. This system can be rearranged into

$$
\left(\tilde{D}_{t} \otimes I_{x}\right) \mathbf{U}+\left(I_{t} \otimes \tilde{D}_{x}\right) \mathbf{U}=\left(P_{t}^{-1} e_{0 t}^{T}\right) \otimes F+G \otimes\left(P_{x}^{-1} e_{0 x}^{T}\right),
$$

where $F=f(\mathbf{x}), G=g(\mathbf{t})$, and $\tilde{D}_{t}, \tilde{D}_{x}$ are of the form (3.4) with $\sigma=-1$. The restrictions of the fully discrete solution $\mathbf{U}$ to the boundaries $x=0, x=1$, $t=0$ and $t=1$ of the computational domain are defined by

$$
\begin{aligned}
\mathbf{U}_{0 t} & =\left(e_{0 t} \otimes I_{x}\right) \mathbf{U}, \quad \mathbf{U}_{N_{t}, t}=\left(e_{N_{t}, t} \otimes I_{x}\right) \mathbf{U} \\
\mathbf{U}_{0 x} & =\left(I_{t} \otimes e_{0 x}\right) \mathbf{U}, \quad \mathbf{U}_{N_{x}, x}=\left(I_{t} \otimes e_{N_{x}, x}\right) \mathbf{U} .
\end{aligned}
$$

The energy method applied to (3.11) now yields

$$
\left\|\mathbf{U}_{N_{t}, t}\right\|_{P_{x}}^{2}=\|F\|_{P_{x}}^{2}+\|G\|_{P_{t}}^{2}-\left\|\mathbf{U}_{N_{x}, x}\right\|_{P_{t}}^{2}-\left\|\mathbf{U}_{0 t}-F\right\|_{P_{x}}^{2}-\left\|\mathbf{U}_{0 x}-G\right\|_{P_{t}}^{2}
$$

which mimics the time integrated version of the continuous energy estimate (2.3), given by

$$
\int_{0}^{1} u(x, 1)^{2} d x=\int_{0}^{1} f(x)^{2} d x+\int_{0}^{1} g(t)^{2} d t-\int_{0}^{1} u(1, t)^{2} d t .
$$




\subsection{Weak interface conditions}

Stable interface conditions were derived using the SAT technique in [4], allowing for provably stable multi-block discretizations where several smaller subdomains are patched together with SAT conditions. This gives added geometric flexibility and can also be used to define an alternative element based approach even in areas where the geometry is simple.

Consider a two-domain formulation of (2.2) on SBP-SAT form. The two grids are defined by $\mathbf{x}=\left(0=x_{0}, \ldots, x_{N_{L}}\right)^{T}$ and $\mathbf{y}=\left(y_{0}, \ldots, y_{N_{R}}=1\right)^{T}$, such that $x_{N_{L}}=y_{0}$, together with corresponding discrete solution vectors $U$ and $V$. Continuity across the interface is imposed using weak SAT penalty conditions as

$$
\begin{aligned}
& U_{t}+D_{L} U=P_{L}^{-1}\left(\sigma_{L} e_{L, N_{L}}^{T}\left(U_{N_{L}}-V_{0}\right)-e_{L, 0}^{T}\left(U_{0}-g(t)\right)\right) \\
& V_{t}+D_{R} V=P_{R}^{-1}\left(\sigma_{R} e_{R, 0}^{T}\left(V_{0}-U_{N_{L}}\right)\right),
\end{aligned}
$$

where $D_{L}$ and $D_{R}$ are SBP operators, and $\sigma_{L}, \sigma_{R}$ are penalty coefficients for the interface treatment. The full solution is given by $W=\left(U^{T}, V^{T}\right)^{T}=$ $\left(W_{0}, \ldots, W_{N}\right)$, where $N=N_{L}+N_{R}+1$. The complete system of ordinary differential equations corresponding to (3.12) can now be written as

$$
W_{t}+\tilde{D} W=\tilde{g}(t)
$$

where

$$
\tilde{D}=P^{-1}\left(\left(\begin{array}{cc}
Q_{L}+e_{L, 0}^{T} e_{L, 0} & 0 \\
0 & Q_{R}
\end{array}\right)-E^{T} \Sigma E\right), \quad \tilde{g}(t)=\left(\begin{array}{c}
P_{L}^{-1} g(t) e_{L, 0}^{T} \\
0
\end{array}\right) .
$$

We have also used the following notation

$$
P=\left(\begin{array}{cc}
P_{L} & 0 \\
0 & P_{R}
\end{array}\right), \quad E=\left(\begin{array}{cc}
e_{L, N_{L}} & 0 \\
0 & e_{R, 0}
\end{array}\right), \quad \Sigma=\left(\begin{array}{cc}
\sigma_{L} & -\sigma_{L} \\
-\sigma_{R} & \sigma_{R}
\end{array}\right) .
$$

Note that $E$ restricts the solution to both interface points, while $E^{T}$ works to impose the weak conditions at the same points.

It is instructive to consider the matrix $\tilde{D}$ in (3.13) as a difference operator acting on the whole spatial domain, augmented with a weak homogeneous boundary condition as in the one-domain case. This point of view was adopted in paper VI, and can be used to simplify the analysis. An investigation into the stability of (3.14) yields

$$
P \tilde{D}+\tilde{D}^{T} P=\operatorname{diag}(1,0, \ldots, 0,1)+E^{T} M E
$$

where

$$
M=\left(\begin{array}{cc}
1 & 0 \\
0 & -1
\end{array}\right)-\left(\Sigma+\Sigma^{T}\right)
$$

The eigenvalues of $\tilde{D}$ have non-negative real parts if the symmetric matrix $M$ in (3.16) is positive semi-definite. For stability, one has to choose $\Sigma$ in such a way that this holds. 


\subsubsection{Conservation and stability}

Closely related to the stability of (3.13) is the concept of conservation across interfaces. This additional property is also needed to capture non-smooth phenomena such as shocks. Consider the general, possibly non-linear, conservation law

$$
u_{t}+\tilde{f}_{x}=0 .
$$

If $\tilde{f}$ is non-smooth, a variational formulation can instead be considered, obtained by multiplying the equation with a smooth test function $\phi$ and then integrating over the domain. By using (2.1) and assuming that $\phi$ vanishes at the domain boundaries, this leads to the weak form

$$
\int_{0}^{t}\left(\left(\phi_{t}, u\right)+\left(\phi_{x}, \tilde{f}\right)\right) d \tau=\left.(\phi, u)\right|_{0} ^{t} .
$$

where $(\cdot, \cdot)$ denotes the $L_{2}$ scalar product. Discretizing with the same operator $\tilde{D}$ as in (3.13) and ignoring boundary data yields

$$
W_{t}+\tilde{D} \tilde{F}=0 .
$$

Recall that $\tilde{D}$ can be viewed as a difference operator acting on the the whole combined spatial grid. Now consider the restriction $\Phi=\phi(\mathbf{x})$ of a smooth function $\phi$ to the grid. The discrete weak form of the conservation law is obtained by multiplying the discrete system with $\Phi^{T} P$, applying (3.15) and integrating in time, which gives

$$
\int_{0}^{t}\left(\left(\Phi_{t}, \tilde{D}\right)_{P}+(\tilde{D} \Phi, \tilde{F})\right)_{P} d \tau=\left.(\Phi, U)_{P}\right|_{0} ^{t}+\int_{0}^{t}(E \Phi)^{T} M(E \tilde{F}) d \tau
$$

where $(\cdot, \cdot)_{P}$ is the scalar product induced by $P$. Note that, since the function $\phi$ is assumed to be smooth, it obtains the same value at both sides of the interface, i.e. $E \Phi=\left(\phi\left(x_{I}\right), \phi\left(x_{I}\right)\right)^{T}$. Thus, the scheme is conservative at the interface if the symmetric matrix $M$ in (3.16) satisfies

$$
M 1=0,
$$

where $\mathbf{1}=(1,1)^{T}$.

We have seen that conservation and energy stability follows if the matrix $M$ (3.16) is positive and satisfies the additional condition (3.17). Both of these properties are achieved with a choice of penalty parameters given by

$$
\sigma_{L}=\sigma+\frac{1}{2}, \quad \sigma_{R}=\sigma-\frac{1}{2}, \quad \sigma \geq 0
$$

With the choice in (3.18), we apply the energy method to (3.13) by multiplying with $W^{T} P$ and adding the transpose. This gives, using (3.15),

$\frac{\partial}{\partial t}\|W\|_{P}^{2}=-W^{T}\left(P \tilde{D}+\tilde{D}^{T} P\right) W+2 W_{0} g=-W_{0}^{2}-W_{N}^{2}-2 \sigma\left(U_{N_{L}}-V_{0}\right)^{2}+2 W_{0} g$. 
Adding and subtracting $g^{2}$ finally yields the energy estimate

$$
\frac{\partial}{\partial t}\|W\|_{P}^{2}=g^{2}-W_{N}^{2}-\left(W_{0}-g\right)^{2}-2 \sigma\left(U_{N_{L}}-V_{0}\right)^{2},
$$

which mimics the one-domain energy estimate (3.6), with an additional damping term from the interface treatment if $\sigma>0$. 


\section{Summary of papers}

\subsection{Paper I}

The basic theoretical properties of the SBP-SAT technique for time integration was investigated. The focus was on time-dependent PDE's discretized in both time and space, where the spatial part of the discretization is stiff. More general initial value problems were also considered, with focus on the question of existence of solutions. To illustrate the general properties, consider a linear initial value problem on the form

$$
\begin{aligned}
U_{t}+A U & =F(t), \quad t \in(0,1) \\
U(0) & =f .
\end{aligned}
$$

The SBP-SAT discretization of (4.1) is given by

$$
\left(D \otimes I_{A}\right) \mathbf{U}+\left(I_{t} \otimes A\right) \mathbf{U}=\left(P^{-1} \sigma e_{0}^{T}\right) \otimes\left(\mathbf{U}_{0}-f\right)+\mathbf{F},
$$

where $\mathbf{U}_{0}=\left(e_{0} \otimes I_{A}\right) \mathbf{U}$, and $\mathbf{F}=\left(F(0)^{T}, F\left(t_{1}\right)^{T}, \ldots, F\left(t_{N-1}\right)^{T}, F(1)^{T}\right)^{T}$. The resulting linear system becomes

$$
\tilde{B} \mathbf{U}=\tilde{\mathbf{c}}
$$

where

$$
\begin{aligned}
\tilde{B} & =\tilde{D} \otimes I_{A}+I_{t} \otimes A \\
\tilde{\mathbf{c}} & =-\left(P^{-1} \sigma e_{0}^{T}\right) \otimes f+\mathbf{F} .
\end{aligned}
$$

The modified difference operator $\tilde{D}$ is defined in (3.4), and the system matrix $\tilde{B}$ in (4.2) is recognized as the Kronecker sum of the two matrices $\tilde{D}$ and $A$. Thus, the eigenvalues of $\tilde{B}$ are given by $\lambda_{\tilde{B}}=\lambda_{\tilde{D}}+\lambda_{A}$, where $\lambda_{\tilde{D}}$ is an eigenvalue to $\tilde{D}$, and $\lambda_{A}$ is an eigenvalue to $A$. Now assume that $\operatorname{Re}\left(\lambda_{A}\right) \geq 0$, i.e. there is no non-physical growth if $A$ is used to model an energy conserving system. If in addition $\operatorname{Re}\left(\lambda_{\tilde{D}}\right)>0$ for all eigenvalues to $\tilde{D}$, then it follows that $\lambda_{\tilde{B}}$ lies strictly in the right half plane, and hence that $\tilde{B}$ is invertible. The additional condition $\operatorname{Re}\left(\lambda_{\tilde{D}}\right)>0$ was validated for several types of high order finite difference operators on SBP form, and is assumed to hold for all SBP operators provided that $\sigma<-1 / 2$. 
The accuracy properties of the SBP-SAT approach in time were also investigated numerically. We found, even when using diagonal norm operators satisfying the accuracy conditions in (2.12), that the accuracy of the temporal scheme is $2 p$ with the choice $\sigma=-1$, which corresponds to the accuracy of the interior finite difference stencils. In the presence of significant stiffness, the order of convergence was reduced to $p$ in some of the test cases.

\subsection{Paper II}

The theory for the SBP-SAT time integration method introduced in paper I was revisited and extended, and several new theoretical results involving stability and accuracy were derived. For example, the stabilty properties for the general linear system (4.1) can be easily demonstrated here. Assume that there exists a positive symmetric matrix $H$ such that $H A+A^{T} H \geq 0$ in (4.1), which implies that the eigenvalues of $A$ have non-negative real parts. Multiplying (4.2) with $\mathbf{U}^{T}(P \otimes H)$ and adding the transpose yields, assuming $\mathbf{F}(t)=0$,

$$
\left\|\mathbf{U}_{N}\right\|_{H}^{2}+\mathbf{U}^{T}\left(P \otimes\left(H A+A^{T} H\right)\right) \mathbf{U}=\|f\|_{H}^{2}-\left\|\mathbf{U}_{0}-f\right\|_{H}^{2},
$$

which is a direct proof showing that $\left\|\mathbf{U}_{N}\right\|_{H}^{2} \leq \mid f \|_{H}$, and thus unconditional stability. Moreover, it mimics the corresponding continuous energy estimate.

In analogy with the multi-block formulation in space outlined in section 3.3, a multi-stage formulation of SBP-SAT in time was proposed. Consider the model problem (3.7), divide the time interval into several pieces, and solve a system analogous to (3.9) on each. This yields the sequence of equations

$$
(\tilde{D}+\lambda I) \mathbf{U}^{n+1}=-P^{-1} \sigma e_{0}^{T} \mathbf{U}_{N}^{n},
$$

with the convention $\mathbf{U}_{N}^{0}=f$. Compared to equation (3.9), the initial data has been replaced with the last stage value of the previous solution $\mathbf{U}^{n}$.

A set of formal stability properties was derived for the SBP-SAT class of temporal schemes with the use of energy estimates, including $A$ - and $L$-stability. Two non-linear stability properties could also be proven, namely $B$-stability (preserving a one-sided Lipschitz condition) and time stability (preserving nongrowth of solution energy). The numerical results obtained in paper I could also be confirmed by showing that the order of the methods with $\sigma=-1$ is in general $2 p$ due to the accuracy of the norm $P(2.13)$. In the proof we focused on the scalar model problem (3.7) and employed a technique based on dual consistency previously used to prove superconvergence of functionals for spatial discretizations $[6,1]$. It was also found that the stiff order of convergence drops to the order $p$ of the truncation error (2.12).

We summarize the formal properties derived for SBP-SAT temporal schemes in the table below. 


\begin{tabular}{|c|c|c|c|c|c|}
\hline Type of norm & order & stiff order & L-stab. & B-stab. & time stab. \\
\hline diagonal & $2 p$ & $p$ & yes & yes & yes \\
\hline block & $2 p$ & $2 p-1$ & yes & no & no \\
\hline
\end{tabular}

It is interesting to note that the properties obtained with diagonal norms are identical to those of the Lobatto IIIC class of implicit Runge-Kutta methods. In a later work [2], it was confirmed that Lobatto IIIC is in fact equivalent to a family of SBP-SAT temporal schemes, employing spectral element SBP operators based on Gauss-Legendre-Lobatto collocation.

\subsection{Paper III}

An efficiency study for implicit temporal schemes was carried out with the purpose of gaining insights into the performance of SBP-SAT in time for realistic applications. As a model problem we used a linear advection-diffusion problem in two dimensions with a boundary layer. Well-posed boundary conditions were imposed using data from a manufactured solution. An explicit, low storage pseudo-time stepping scheme with multigrid accelleration was employed to converge the solutions.

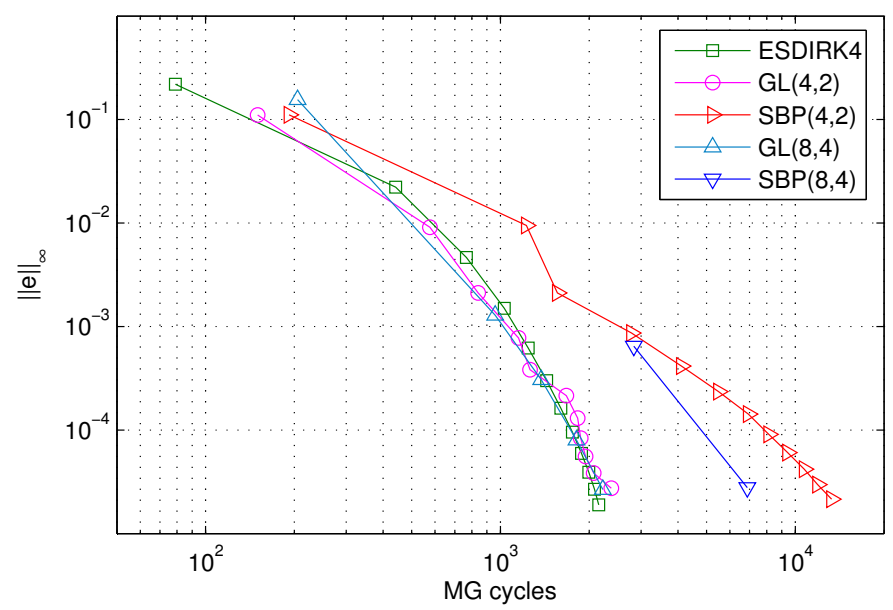

Figure 4.1: Convergence as a function of total work, defined as the sum of multi-grid cycles over all implicit stages.

The main finding was that the multigrid convergence deteriorates for classical SBP-SAT schemes based on finite difference schemes, which is probably due to a combination of the spectral properties and the large number of stages in each 
solve. The results in terms of multigrid efficiency can be seen in Figure 4.1 for a collection of methods, including SBP-SAT schemes of both finite difference and spectral type, as well as a popular diagonal implicit Runge-Kutta method for comparison. It is interesting to note that, except for the classical finite difference schemes, the efficiency is almost identical for all other methods.

\subsection{Paper IV}

The SBP-SAT technique in time was extended to include problems with second order derivatives in time. The following scalar test problem was considered

$$
\begin{gathered}
u_{t t}+\alpha^{2} u_{t}+\beta^{2}=0, \quad t \in(0,1) \\
u=f, \quad u_{t}=g, \quad t=0 .
\end{gathered}
$$

The energy method for this problem is applied by multiplying with $u_{t}$ and integrating in time, giving

$$
u_{t}(1)^{2}+\beta^{2} u(1)^{2}+2 \alpha^{2}\left\|u_{t}\right\|^{2}=g^{2}+\beta^{2} f^{2} .
$$

A wide stencil approximation is obtained by applying a first derivative SBP operator twice, which results in the system

$$
\tilde{D}\left(\tilde{D} U+P^{-1} \sigma f e_{0}^{T}\right)+\alpha^{2}\left(\tilde{D} U+P^{-1} \sigma f e_{0}^{T}\right)+\beta^{2} U=-P^{-1} \sigma g e_{0}^{T},
$$

where $\tilde{D}$ is defined as in (3.4). The discrete energy method for $\sigma=-1$ gives

$$
\left(\tilde{U}_{t}\right)_{N}^{2}+\beta^{2} U_{N}^{2}+\alpha^{2} \tilde{U}_{t}^{T} P_{t} \tilde{U}_{t}=g^{2}+\beta^{2} f^{2}-\left(\left(\tilde{U}_{t}\right)_{0}-g\right)^{2}-\left(U_{0}-f\right)^{2},
$$

where we have used a modified first derivative approximation augmented with the weak initial condition: $\tilde{U}_{t}=D U+P^{-1} e_{0}^{T}\left(U_{0}-f\right)$. The new technique was also applied to the wave equation, including the case with boundary conditions involving derivatives in both space and time, specifically in the form of the first order non-reflecting conditions of Engquist \& Majda [5].

The possibility of using compact second derivative stencils was also investigated. The discrete second derivative operator obtained by applying the first derivative operator twice can be written on the form

$$
D^{2}=P^{-1}\left(-D^{T} P D+\left(-e_{0}^{T} e_{0}-e_{N}^{T} e_{N}\right) D\right) .
$$

We considered the compatible formulation of compact SBP operators, developed in $[13,11]$ for discretizations of second order spatial derivatives. Starting from the wide operator and adding a correction term, the compact operator can be written on the general form

$$
D_{c}=P^{-1}\left(-D^{T} P D-R+\left(-e_{0}^{T} e_{0}+e_{N}^{T} e_{N}\right) D\right) .
$$


For stable compact spatial discretizations, the correction term must satisfy $R+R^{T} \geq 0$, whereas a temporal compact operator needs to satisfy the two conditions

$$
D^{T} R+R D \leq 0, \quad e_{0}^{T} R=0 .
$$

Unfortunately, the conclusion was that compact stencil operators for time integration satisfying these properties can most likely not be constructed. This conclusion was drawn based on a combination of analysis and use of mathematical software, focusing on a second order accurate compact stencil with a boundary closure expressed on a general form.

\subsection{Paper V}

The accuracy of an interface treatment in two dimensions with a non-conforming grid interface was considered. A stable procedure for such interfaces was derived in [12], employing so-called SBP preserving interpolation operators. The interpolation operators presented therein as well as in later works had a lower order formal accuracy than that of the SBP operators themselves. Moreover, the numerical results presented in the literature had so far been ambigious as to whether this drop would affect the convergence rate of the solution. The main contribution to this development from paper $\mathrm{V}$ consists of a formal proof explaining the previously reported reduced accuracy of the interpolation operators.
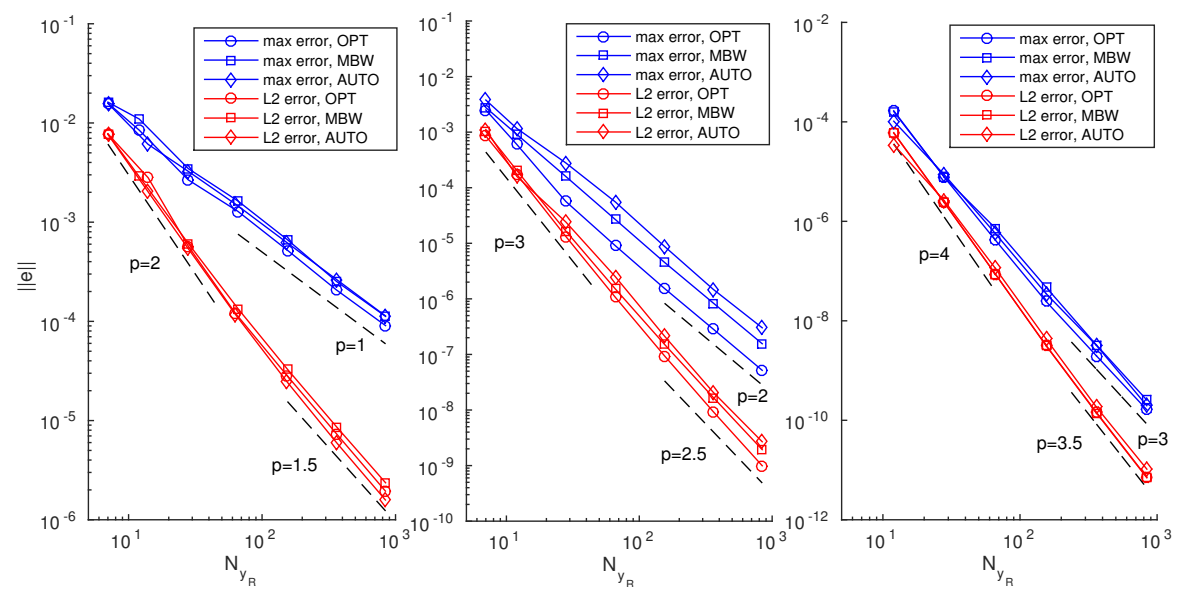

Figure 4.2: Rates of convergence in both maximum and $L_{2}$ norm for three different types of SBP preserving interpolation operators. The three figures show the results from left to right using 2 :nd, 4 :th and 6 :th order finite difference stencils in the interior, respectively 
Consider a two-dimensional advection model problem on a cartesian domain, given by

$$
u_{t}+a u_{x}+b u_{y}=0, \quad(x, y) \in(0,1)^{2} .
$$

The stable and conservative two-domain SBP-SAT discretization of (4.4) derived in [12] for a non-conforming vertical grid interface can be written as, ignoring the exterior boundary conditions,

$$
\begin{aligned}
U_{t}+a\left(D_{\mathbf{x}_{L}} \otimes I_{\mathbf{y}_{L}}\right) U+b\left(I_{\mathbf{x}_{L}} \otimes D_{\mathbf{y}_{L}}\right) U & =\frac{1}{2} P_{\mathbf{x}_{L}}^{-1} e_{\mathbf{x}_{L}, N_{L}}^{T} \otimes\left(U_{I}-\tilde{\mathcal{P}} V_{I}\right) \\
V_{t}+a\left(D_{\mathbf{x}_{R}} \otimes I_{\mathbf{y}_{R}}\right) V+b\left(I_{\mathbf{x}_{R}} \otimes D_{\mathbf{y}_{R}}\right) V & =-\frac{1}{2} P_{\mathbf{x}_{R}}^{-1} e_{\mathbf{x}_{R}, 0}^{T} \otimes\left(V_{I}-\mathcal{P} U_{I}\right),
\end{aligned}
$$

where $U_{I}=\left(e_{\mathbf{x}_{L}, N_{L}} \otimes I_{\mathbf{y}_{L}}\right) U$ and $V_{I}=\left(e_{\mathbf{x}_{R}, 0} \otimes I_{\mathbf{y}_{R}}\right) V$ both represent the solution along the common grid interface, while $\mathcal{P}$ and $\tilde{\mathcal{P}}$ are interpolation operators. This formulation generalizes the non-dissipative choice $\sigma=0$ of the one-dimensional interface problem in (3.18). The SBP preserving condition sufficient for stability is

$$
P_{\mathbf{y}_{L}} \tilde{\mathcal{P}}=\mathcal{P}^{T} P_{\mathbf{y}_{R}}
$$

A proof was presented showing that (4.6) leads to a suboptimal accuracy bound on $\mathcal{P}$ and $\tilde{\mathcal{P}}$ compared to the accuracy (2.12) of the SBP operators. The truncation error of the scheme (4.5) is locally reduced by one order as a result. Numerical investigations confirmed the resulting drop in convergence by one order in maximum norm, and by a half order in $L_{2}$ norm. Interestingly however, these reduced convergence rates only became clearly visible at very low error levels. The convergence results can be seen in Figure 4.2.

\subsection{Paper VI}

A new framework for expressing SBP-SAT discretizations on completely general multi-block, multi-element or hybrid grids was presented. This involves the introduction of multi-domain SBP operators in several spatial dimensions, satisfying a generalized SBP property. Completely general discretizations involving multiple numerical interfaces can thus be expressed within a single compact formulation. The generality of the new framework was demonstrated by showing that it conforms to tensor product extensions of one-dimensional SBP operators in curvilinear coordinates, as well as to the standard multi-dimensional nodal dG method.

Consider a continuous multi-dimensional hyperbolic model problem on the form

$$
\begin{aligned}
& u_{t}+\mathbf{a} \cdot \nabla u=0, \quad x \in \Omega, \quad t>0 \\
& u=g(x, t), \quad x \in \Gamma^{-}, \quad t>0 \\
& u=f, \quad x \in \Omega, \quad t=0 \text {. }
\end{aligned}
$$


In (4.7), $\Gamma^{-}$denotes the inflow part of the domain boundary defined by the condition $\mathbf{a} \cdot \mathbf{n}<0$, where $\mathbf{n}$ is the outward pointing normal. The IBP property in several dimensions is given by

$$
(\phi, \mathbf{a} \cdot \nabla \psi)=\oint_{\Gamma} \phi \psi(\mathbf{a} \cdot \mathbf{n}) d s-(\mathbf{a} \cdot \nabla \phi, \psi)
$$

The continuous energy estimate is given by

$$
\|u\|_{t}^{2}=\|g\|_{\text {in }}^{2}-\|u\|_{\text {out }}^{2},
$$

which follows from employing the energy method to (4.7), and using (4.8). The two semi-norms on $\Gamma^{+}$and $\Gamma^{-}$respectively are defined as

$$
\|u\|_{\text {out }}^{2}=\oint_{\Gamma^{+}}|u|^{2}(\mathbf{a} \cdot \mathbf{n}) d s, \quad\|u\|_{\text {in }}^{2}=-\oint_{\Gamma^{-}}|u|^{2}(\mathbf{a} \cdot \mathbf{n}) d s .
$$

A generalized SBP property for discrete operators approximating the hyperbolic operator $\mathbf{a} \cdot \nabla$ on multiple domains was presented. After introducing a set of restriction and discrete boundary integration operators, each one acting on a part of the domain boundary, the SBP property was formulated as

$$
Q+Q^{T}=\sum_{i=1}^{n} E_{\Gamma_{i}}^{T} P_{\Gamma_{i}} \Lambda_{\Gamma_{i}} E_{\Gamma_{i}},+E^{T} M E
$$

where $M \geq 0$, and where $\Lambda_{\Gamma_{i}}$ are diagonal matrices with the values of $\mathbf{a} \cdot \mathbf{n}$ inserted on the diagonal. The resulting discrete energy estimate becomes

$$
\begin{aligned}
\frac{d}{d t}\|U\|_{P}^{2} & =\sum_{i=1}^{n}\left(\left\|G_{\Gamma_{i}}(t)\right\|_{I N}^{2}-\left\|U_{\Gamma_{i}^{-}}\right\|_{O U T}^{2}-\left\|U_{\Gamma_{i}^{-}}-G_{\Gamma_{i}}(t)\right\|_{I N}^{2}\right) \\
& -(E U)^{T} M(E U),
\end{aligned}
$$

where $E U$ denotes the restriction of the solution to the interfaces between the subdomains. The estimate (4.12) mimics the continuous one with extra dissipation from the weak boundary and interface conditions. We derived new theoretical results establishing the interrelations between accuracy, conservation and energy stability for such formulations. Based on these results, a generalized description of SBP preserving interpolation operators was also formulated. 


\section{References}

[1] J. Berg and J. Nordström. Superconvergent functional output for timedependent problems using finite differences on summation-by-parts form. Journal of Computational Physics, 231(20):6846-6860, 2012.

[2] P. Boom and D. Zingg. Runge-kutta characterization of the generalized summation-by-parts approach in time. 2014. Submitted (preprint: http: //arxiv.org/abs/1410.0202).

[3] M. H. Carpenter, D. Gottlieb, and S. Abarbanel. Time-stable boundary conditions for finite-difference schemes solving hyperbolic systems: Methodology and application to high-order compact schemes. Journal of Computational Physics, 111(2):220-236, 1994.

[4] M. H. Carpenter, J. Nordström, and D. Gottlieb. A stable and conservative interface treatment of arbitrary spatial accuracy. Journal of Computational Physics, 148:341-365, 1999.

[5] B. Engquist and A. Majda. Absorbing boundary conditions for the numerical simulation of waves. Mathematics of Computations, 139(31):629-651, 1977.

[6] J. E. Hicken and D. W. Zingg. Superconvergent functional estimates from summation-by-parts finite-difference discretizations. SIAM Journal on Scientific Computing, 33(2):893-922, 2011.

[7] J. E. Hicken and D. W. Zingg. Summation-by-parts operators and high order quadrature. Journal of Computational and Applied Mathematics, 237(1):111-125, 2013.

[8] H.-O. Kreiss and G. Scherer. Finite element and finite difference methods for hyperbolic partial differential equations, in: C. De Boor (Ed.), Mathematical Aspects of Finite Elements in Partial Differential Equation. Academic Press, New York, 1974.

[9] P. Lax and Richtmyer. Survey of the stability of linear finite difference equations. Communications on pure and applied mathematics, 9:267-293, 1956.

[10] P. Lax and B. Wendroff. Systems of conservation laws. Communications on pure and applied mathematics, 13:217-237, 1960.

[11] K. Mattsson. Summation by parts operators for finite difference approximations of second-derivatives with variable coefficients. Journal of Scientific Computing, 51(3):650-682, 2012.

[12] K. Mattsson and M. H. Carpenter. Stable and accurate interpolation operators for high-order multiblock finite difference methods. SIAM Journal on Scientific Computing, 32:2298-2320, 2010. 
[13] K. Mattsson, M. Svärd, and M. Shoeybi. Stable and accurate schemes for the compressible Navier-Stokes equations. Journal of Computational Physics, 227:2293-2316, 2008.

[14] J. Nordström. Conservative finite difference formulations, variable coefficients, energy estimates and artificial dissipation. Journal of Scientific Computing, 29(3):375-404, 2006.

[15] M. Svärd. On coordinate transformations for summation-by-parts operators. Journal of Scientific Computing, 20(1):29-42, 2004. 


\section{Papers}

The articles associated with this thesis have been removed for copyright reasons. For more details about these see:

http://urn.kb.se/resolve?urn=urn:nbn:se:liu:diva-126172 